Check for updates

Cite this: RSC Adv., 2017, 7, 51919

Received 5th September 2017 Accepted 20th October 2017

DOI: $10.1039 / c 7 r a 09868 \mathrm{e}$

rsc.li/rsc-advances

\section{A highly efficient gas-dominated and water- resistant flame retardant for non-charring polypropylene}

\author{
Zhu-Bao Shao, (D) a Ming-Xin Zhang, ${ }^{a}$ Ye Han, ${ }^{\text {*a }}$ Xu-Dong Yang, ${ }^{a} \mathrm{Jing}_{\mathrm{Jin}}{ }^{\mathrm{a}}$ \\ and Rong-Kun Jian*b
}

Traditional phosphorus-nitrogen (P-N) flame-retardant systems for polypropylene (PP) always act through joint action of the gaseous phase and condensed phase, and are accompanied with a decrease of the thermal stability and water resistance. In this work, a novel mono-component and gas-dominated flame retardant, named DPPIP, was prepared through an amidation reaction of diphenylphosphinyl chloride and piperazine, and used to flame retard PP. Experimental results confirmed that both the thermal stability and water resistance of PP/DPPIP were improved. The initial thermal decomposition temperature of PP/25 wt\% DPPIP at $5 \mathrm{wt} \%$ weight loss was $287.5{ }^{\circ} \mathrm{C}$ under air atmosphere, which is higher than that of neat PP $\left(266.1^{\circ} \mathrm{C}\right)$. Besides, a water-resistance test verified that PP/25 wt\% DPPIP had a weight loss of only about $0.52 \mathrm{wt} \%$. More importantly, the flame retardant ability of PP/25 wt\% DPPIP had been greatly improved, passing the $V-0$ rating (UL-94). Furthermore, after the water-resistance test, the LOI value of PP/25 wt\% DPPIP exhibited nearly no difference and still passed the UL-94 V- 0 rating. A cone calorimeter $(\mathrm{CC})$ result indicated that DPPIP had a positive effect on inhibiting heat release of PP during combustion. All of these combustion tests displayed that there was no char left. The flame retardant mechanism of DPPIP was investigated with py-GC/MS and TG-FTIR. The results illustrated that the gaseous phase resulting from the thermal decomposition of DPPIP played the leading role in the self-extinguishing behavior of PP/DPPIP, which consisted of a large amount of inflammable gaseous products such as piperazine and its derivatives, and phosphorus-containing structures.

\section{Introduction}

Halogen-free flame retardant systems, such as metal hydroxides, metal borates and intumescent flame retardants (IFR) etc., have been utilized to obtain flame-retardant PP composites. ${ }^{\mathbf{1 - 4}}$ Among these halogen-free flame retardant systems, intumescent flame retardants, especially phosphorus-nitrogen $(\mathrm{P}-\mathrm{N})$ flame retardants, have attracted much attention in flameretardant PP due to their high flame-retardant efficiency. ${ }^{5-7}$

In order to obtain better flame-retardant efficiency of PP, a lot of work has been done in past decades, especially research on the flame-retardant mechanism. Generally, traditional P-N flame retardants for PP mainly contain three components, which are an acid source, blowing agent and charring agent, ${ }^{\mathbf{8 , 9}}$ and when $\mathrm{P}-\mathrm{N}$ flame retardants are heated beyond a critical temperature they can generate multicellular swollen chars on the surface of the polymer accompanied by decomposition of the blowing agent, slowing down the heat and oxygen transfer,

${ }^{a}$ Institute of Chemical Engineering, Changchun University of Technology, Changchun 130012, China. E-mail: zhu871227@163.com

${ }^{b}$ Fujian Key Laboratory of Polymer Materials, College of Materials Science and Engineering, Fujian Normal University, Fuzhou 350007, China.E-mail: jrkht1987@fjnu.edu.cn thus protecting the substrate from burning. ${ }^{\mathbf{1 0 , 1 1}}$ Therefore, these flame-retardant systems for PP usually act through a gaseous phase and condensed-phase flame-retardant mechanism together. Compared with the production of gas, the condensedphase formation of $\mathrm{P}-\mathrm{O}-\mathrm{C}$ structures during combustion is one of the methods to improve the flame-retardant efficiency, and the typical flame retardant system is a mixture of ammonium polyphosphate and charring agent (CA). ${ }^{12-14}$ Wang et al. ${ }^{15}$ synthesized a novel charring agent (CA), namely MTEC, and found that a PP/APP/MTEC system could achieve a LOI value of $28.5 \%$ and acquire a UL-94 V-0 rating at $20 \mathrm{wt} \% \mathrm{APP} / \mathrm{CA}$ through the formation of $\mathrm{P}-\mathrm{O}-\mathrm{C}$ structures during combustion. The condensed-phase formation of $\mathrm{P}-\mathrm{N}-\mathrm{C}$ structures during combustion is also an efficient method to enhance the flameretardant efficiency of PP. In our previous papers, ${ }^{\mathbf{1 6 - 1 8}}$ APPs modified with ethylenediamine (EDA), ethanolamine (ETA) or piperazine (PIP) were incorporated alone into PP. The results also indicated that $\mathrm{P}-\mathrm{N}-\mathrm{C}$ structures were formed during combustion, which could enhance the flame-retardant efficiency for PP. In summary, APP is an essential component to facilitate the formation of stable char during two $\mathrm{P}-\mathrm{N}$ flameretardant methods. Unfortunately, the thermal stability and water resistance of APP and modified APPs were not improved 
due to the production of large amounts of amine salts and the introduction of organic amine salts. Although lots of work has been done, such as the modification of APP and microencapsulated APP, ${ }^{13,19}$ these problems still exist, which has limited the application of APP in the practical field. Thus, some researchers have considered whether PP could obtain an excellent efficiency only acting through a gas-dominated flame retardant.

In the present work, a novel mono-component, only gasdominated and $\mathrm{P}-\mathrm{N}$ containing flame retardant, named DPPIP, was synthesized through the reaction of diphenylphosphinyl chloride and piperazine, and different measurements were used to confirm its chemical structure. The thermal stability and water resistance of DPPIP were examined. PP/DPPIP blends were prepared via physical blending, and their thermal stability, water-solubility and flame retardancy were also studied. Finally, the flame retardant mechanism of DPPIP was investigated with the aid of different measurements.

\section{Experimental section}

\subsection{Materials}

Diphenylphosphinyl chloride (DPPC) was supplied by Shanghai Changgen Chemical Reagent Co., Ltd. (Shanghai, China); piperazine (AR), chloroform (AR) and triethylamine (AR) were purchased from Tianjin Kemiou Chemical Reagent Co., Ltd. (Tianjin, China); polypropylene (T30S) was obtained from Petro China Lanzhou Petrochemical Co., Ltd. (Lanzhou, China).

\subsection{Measurements}

Fourier transform infrared spectroscopy (FTIR) tests were performed on a Nicolet FTIR 170SX spectrometer (Nicolet, America) using a $\mathrm{KBr}$ disk, and the wavenumber range was set from 4000-400 $\mathrm{cm}^{-1}$.

${ }^{1} \mathrm{H}$ nuclear magnetic resonance $\left({ }^{1} \mathrm{H}-\mathrm{NMR}\right)$ spectra and ${ }^{31} \mathrm{P}$ nuclear magnetic resonance $\left({ }^{31} \mathrm{P}-\mathrm{NMR}\right)$ spectra were recorded on a Bruker AV II-400 MHz spectrometer (Bruker, Switzerland) using $\mathrm{CDCl}_{3}$ as solvent. The sample (85 mg) was put into an NMR tube, then a solution of $\mathrm{CDCl}_{3}(5 \mathrm{~mL})$ was injected into the NMR tube, and next the NMR tube was shaken until the solids were dissolved in $\mathrm{CDCl}_{3}$. The concentrations of DPPIP, PIP or DPPC were about $1.7 \mathrm{~g} / 100 \mathrm{~mL}$. The NMR test was performed at about $25{ }^{\circ} \mathrm{C}$, and TMS was applied. The peak which appeared at $0 \mathrm{ppm}$ in the ${ }^{1} \mathrm{H}-\mathrm{NMR}$ spectra was ascribed to TMS.

X-ray photoelectron spectroscopy (XPS) spectra were recorded on an XSAM80 spectrometer (Kratos Co, UK) equipped with $\mathrm{Al} \mathrm{K} \alpha$ excitation radiation $(h \nu-1486.6 \mathrm{eV})$.

Tensile testing was completed in accordance with the procedures in GB/T 1040.1-2006 at a crosshead speed of 20 $\mathrm{mm} \mathrm{min}^{-1}$. The Izod impact properties were tested in accordance with the procedures in GB/T 1843-2008 and the depth of the nick was $2 \mathrm{~mm}$.

Thermogravimetric (TG) analysis was carried out on a TG 209 F1 (NETZSCH, Germany) thermogravimetric analyzer at a heating rate of $10{ }^{\circ} \mathrm{C} \min ^{-1}$ under a nitrogen or air flow of $50 \mathrm{~mL} \mathrm{~min}^{-1}$ in the temperature range from 40 to $700{ }^{\circ} \mathrm{C}$.
The LOI value was measured using an HC-2C oxygen index instrument (Jiangning, China) according to ASTM D2863-97. The sheet dimensions of all of the samples were $130 \mathrm{~mm} \times$ $6.5 \mathrm{~mm} \times 3.2 \mathrm{~mm}$.

The UL-94 vertical burning level was tested on a CZF-2 instrument (Jiangning, China) according to ASTM D 3801. The dimensions of the samples were $130 \mathrm{~mm} \times 13 \mathrm{~mm} \times 3.2 \mathrm{~mm}$.

The flammability of the samples was measured on a CC device (Fire Testing Technology, UK). Samples with dimensions of $100 \mathrm{~mm} \times 100 \mathrm{~mm} \times 3 \mathrm{~mm}$ were exposed to a radiant cone at a heat flux of $50 \mathrm{~kW} \mathrm{~m}^{-2}$.

The thermogravimetry-Fourier transform infrared spectroscopy (TG-FTIR) apparatus consisted of a TG 209 F1 (NETZSCH, Germany) thermogravimetric analyzer and a 170 SX FTIR spectrometer (Nicolet, America). A sample (about $6 \mathrm{mg}$ ) was heated at a rate of $10^{\circ} \mathrm{C} \mathrm{min}^{-1}$ in the temperature range from 40 to $700{ }^{\circ} \mathrm{C}$ under a nitrogen flow of $50 \mathrm{~mL} \mathrm{~min}^{-1}$.

The samples for the py-GC/MS tests were first pyrolyzed in a pyrolyzer (CDS5200) under helium atmosphere. The relevant samples $(300 \mu \mathrm{g})$ were heated from ambient temperature to $600{ }^{\circ} \mathrm{C}$ at a rate of $20{ }^{\circ} \mathrm{C} \mathrm{ms}{ }^{-1}$ and kept for $15 \mathrm{~s}$. Then, the volatile products were carried through helium to a Perkin-Elmer Clarus680GC-SQ8MS system coupled with the pyrolyzer. The temperature of the capillary column (HP-5, $0.25 \mathrm{~mm}$ ) of the GC was held at $40{ }^{\circ} \mathrm{C}$ for $3 \mathrm{~min}$; afterward, the temperature was increased to $280{ }^{\circ} \mathrm{C}$ at a heating rate of $10{ }^{\circ} \mathrm{C} \mathrm{min}{ }^{-1}$ and then kept at $280{ }^{\circ} \mathrm{C}$ for $5 \mathrm{~min}$. The injector temperature was maintained at $280{ }^{\circ} \mathrm{C}$. The MS indicator was operated in the electron impact mode at an electron energy of $70 \mathrm{eV}$, and the ion source temperature was kept at $250{ }^{\circ} \mathrm{C}$. The identification of the pyrolysis products was carried out by comparing to the NIST MS library.

The water-resistance of the flame retardant and modified PP was tested according to the following procedure: $10 \mathrm{~g}$ of flame retardant was put into $100 \mathrm{~mL}$ of distilled water at $70{ }^{\circ} \mathrm{C}$ and stirred for $60 \mathrm{~min}$. The suspension was then filtered. Next, the obtained solid was dried to a constant weight at $100{ }^{\circ} \mathrm{C}$. Finally, the solubility of the flame retardant in water was obtained. ${ }^{20}$ The modified PP (the weight of $M_{0}$ ) was put into distilled water at $70{ }^{\circ} \mathrm{C}$, and kept at this temperature for $168 \mathrm{~h}$ (the water was replaced at each interval of $24 \mathrm{~h}$ ). Then the treated specimens were taken out, and dried to a constant weight at $100{ }^{\circ} \mathrm{C}$. The weight of the dried sample is $M_{1}$, so the weight loss can be calculated based on the following equation: $\left[\left(M_{0}-M_{1}\right) / M_{0}\right] \times$ $100 \% .^{20}$

\subsection{Synthesis of DPPIP}

$47 \mathrm{~g}$ of diphenylphosphinyl chloride and $100 \mathrm{~mL}$ of chloroform were mixed in a $250 \mathrm{~mL}$ three-necked round-bottom flask equipped with a reflux condenser, dry $\mathrm{N}_{2}$ inlet, and a dropping funnel. The mixture was agitated in an ice-bath under nitrogen atmosphere. Half an hour later, $8 \mathrm{~g}$ of piperazine dissolved in chloroform $(20 \mathrm{~mL})$ was added over $1 \mathrm{~h}$. Then $60 \mathrm{~g}$ of triethylamine was added to the solution. After completing the addition, the solution was heated to $75{ }^{\circ} \mathrm{C}$ for $8 \mathrm{~h}$ until the reaction completely finished. Finally, the solution was cooled to room 


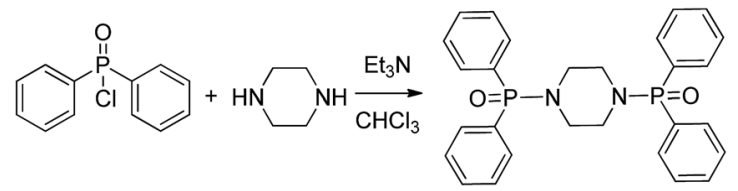

Scheme 1 The synthetic route of DPPIP.

temperature and then filtered. The filtrate was evaporated under reduced pressure to obtain the crude product. The product was washed with water repeatedly to remove the impurities, then dried at $85{ }^{\circ} \mathrm{C}$ for $12 \mathrm{~h}$ under vacuum to a constant weight $(84.3 \%$ yield). The reaction formula is described in Scheme 1.

\subsection{Sample preparation}

DCPIP was dried in a vacuum oven at $85{ }^{\circ} \mathrm{C}$ for $12 \mathrm{~h}$. Then PP blends filled with different ratios of DCPIP were prepared via a twin-screw extruder (CTE 20, Kebeilong Keya Nanjing Machinery Co., Ltd, Nanjing, China) with a rotation speed of $150 \mathrm{rpm}$ with the following temperature protocol from the feed zone to the die: $175,180,190,185,180$ and $170{ }^{\circ} \mathrm{C}$. Finally, the extruded pellets were hot-pressed into different samples by a plate vulcanizer (Qingdao Yadong Rubber Viachinery Co. Ltd. China).

\section{Results and discussion}

\subsection{Characterization of DPPIP}

The FTIR spectrum of DPPIP is shown in Fig. 1. The peaks at around $3025.6-3059.4 \mathrm{~cm}^{-1}$ and $760.9 \mathrm{~cm}^{-1}$ are ascribed to the $\mathrm{C}-\mathrm{H}$ vibrations of the benzene rings. The peaks located at 2948.6-2962.8 $\mathrm{cm}^{-1}$ are assigned to the $\mathrm{C}-\mathrm{H}$ stretching vibrations of PIP. The peaks at $1116.4 \mathrm{~cm}^{-1}$ and $722.5 \mathrm{~cm}^{-1}$ correspond to the $\mathrm{P}-\mathrm{N}-\mathrm{C}$ vibrations, ${ }^{16-18}$ and the peak at $1178.8 \mathrm{~cm}^{-1}$ is assigned to the $\mathrm{P}=\mathrm{O}$ vibration. The results show that DPPIP had been prepared successfully.

To confirm the formation of DPPIP, ${ }^{1} \mathrm{H}$-NMR and ${ }^{31} \mathrm{P}$-NMR tests of DPPC, PIP and DPPIP were performed, and the results

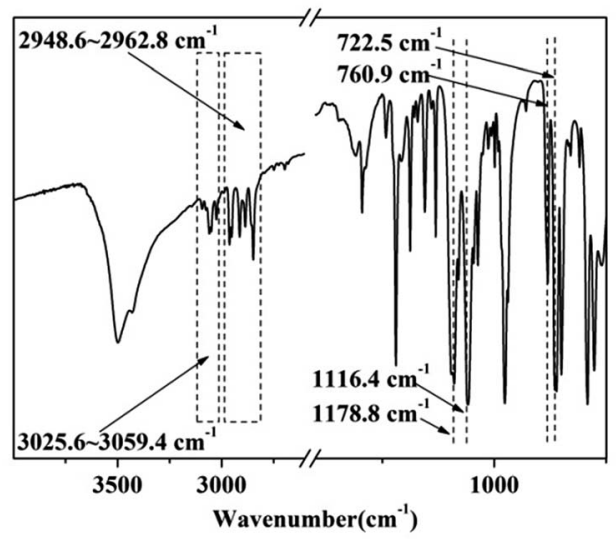

Fig. 1 FTIR spectrum of DPPIP. are displayed in Fig. 2. All of the protons can be attributed to the expected signals. Compared with the ${ }^{1} \mathrm{H}$-NMR spectrum of PIP (Fig. 2b), the $\mathrm{N}-\mathrm{H}$ signal at $1.75 \mathrm{ppm}$ disappeared and the peak at $2.80 \mathrm{ppm}$ shifted to $3.11 \mathrm{ppm}$ in DPPIP, which is assigned to $-\mathrm{CH}_{2}-$ in the $-\mathrm{CH}_{2}-\mathrm{N}-\mathrm{P}-$ group, indicating that the $\mathrm{P}-\mathrm{N}$ bond is formed. In the ${ }^{31} \mathrm{P}$-NMR spectra, there was only one peak at $44.95 \mathrm{ppm}$ for DPPC, which is ascribed to the $\mathrm{P}$ in the $-\mathrm{P}-\mathrm{Cl}$ group, and DPPIP displayed one characteristic peak at $29.47 \mathrm{ppm}$, indicating that the $\mathrm{P}-\mathrm{N}$ bond is formed and a high purity of DPPIP. All of the ${ }^{1} \mathrm{H}-\mathrm{NMR}$ and ${ }^{31} \mathrm{P}$-NMR results demonstrate that DPPIP was obtained successfully.

To further determine the structure of DPPIP, XPS measurements of PIP and DPPIP were carried out. Their $\mathrm{N}_{1 \mathrm{~s}}$ spectra are shown in Fig. 3. For PIP, the peak at $399.2 \mathrm{eV}$ is ascribed to the $\mathrm{N}$ in $-\mathrm{C}-\mathrm{NH}-\mathrm{C}-.^{17}$ While for DPPIP, a new peak at $402.0 \mathrm{eV}$ is attributed to the $\mathrm{N}$ in $-\mathrm{P}-\mathrm{N}-\mathrm{C}-$, further indicating that DPPIP was formed.

\subsection{Water resistance}

Water resistance is an important property to assess the usability of flame retardants in polymers. Thus, the water solubility of DPPIP was tested and compared with our previously modified APPs (PA-APP), and the corresponding data are listed in Table 1. It could be observed that PA-APP is easily dissolved in $70{ }^{\circ} \mathrm{C}$ water due to the large amount of hydrophilic amine salt, and the water solubility value is $10.0 \mathrm{~g} / 100 \mathrm{~mL}$ of water. However, because of the hydrophobic nature of $\mathrm{P}-\mathrm{N}$ and the benzene rings, the water solubility of DPPIP is less than $0.01 \mathrm{~g} / 100 \mathrm{~mL}$ of water. Moreover, the water resistance of PP/DPPIP and PP/PAAPP was also evaluated through water-solubility experiments, and the results are listed in Table 1. For PP/DPPIP, the weight loss is only $0.52 \mathrm{wt} \%$, which is far less than that for PP/PA-APP. These results demonstrate that DPPIP and PP/DPPIP have an excellent water resistance.

\subsection{Thermogravimetric analysis}

TG and derivative thermogravimetric (DTG) curves of neat PP, DPPIP and PP/DPPIP under $\mathrm{N}_{2}$ and air atmosphere are illustrated in Fig. 4, and the corresponding data are listed in Table 2. There is only one degradation step for DPPIP ranging from 300$420{ }^{\circ} \mathrm{C}$ under $\mathrm{N}_{2}$ atmosphere, and the initial decomposition temperature $\left(T_{5 \%}\right.$, defined as the temperature where the mass loss is $5 \mathrm{wt} \%$ ) of DPPIP is $328.9^{\circ} \mathrm{C}$, which is lower than that of neat PP $\left(383.5^{\circ} \mathrm{C}\right)$. Consequently, with the addition of DPPIP, the initial decomposition temperature of modified PP $\left(\mathrm{N}_{2}\right)$ decreased, and it corresponds well to that in the calculated curve. However, the degradation of PP/DPPIP under air atmosphere is very different, as the initial decomposition temperature of DPPIP $\left(310.8^{\circ} \mathrm{C}\right)$ is higher than that of neat PP $\left(266.1^{\circ} \mathrm{C}\right)$. As shown in Fig. 4c and d, with the temperature rising, the residues at $700{ }^{\circ} \mathrm{C}$ are almost gone for the neat PP, DPPIP and PP/DPPIP both under $\mathrm{N}_{2}$ and air atmosphere. The TG results suggest that the char layer is not formed at $700{ }^{\circ} \mathrm{C}$, indicating that DPPIP generated almost only gas-phase products during the decomposition. 

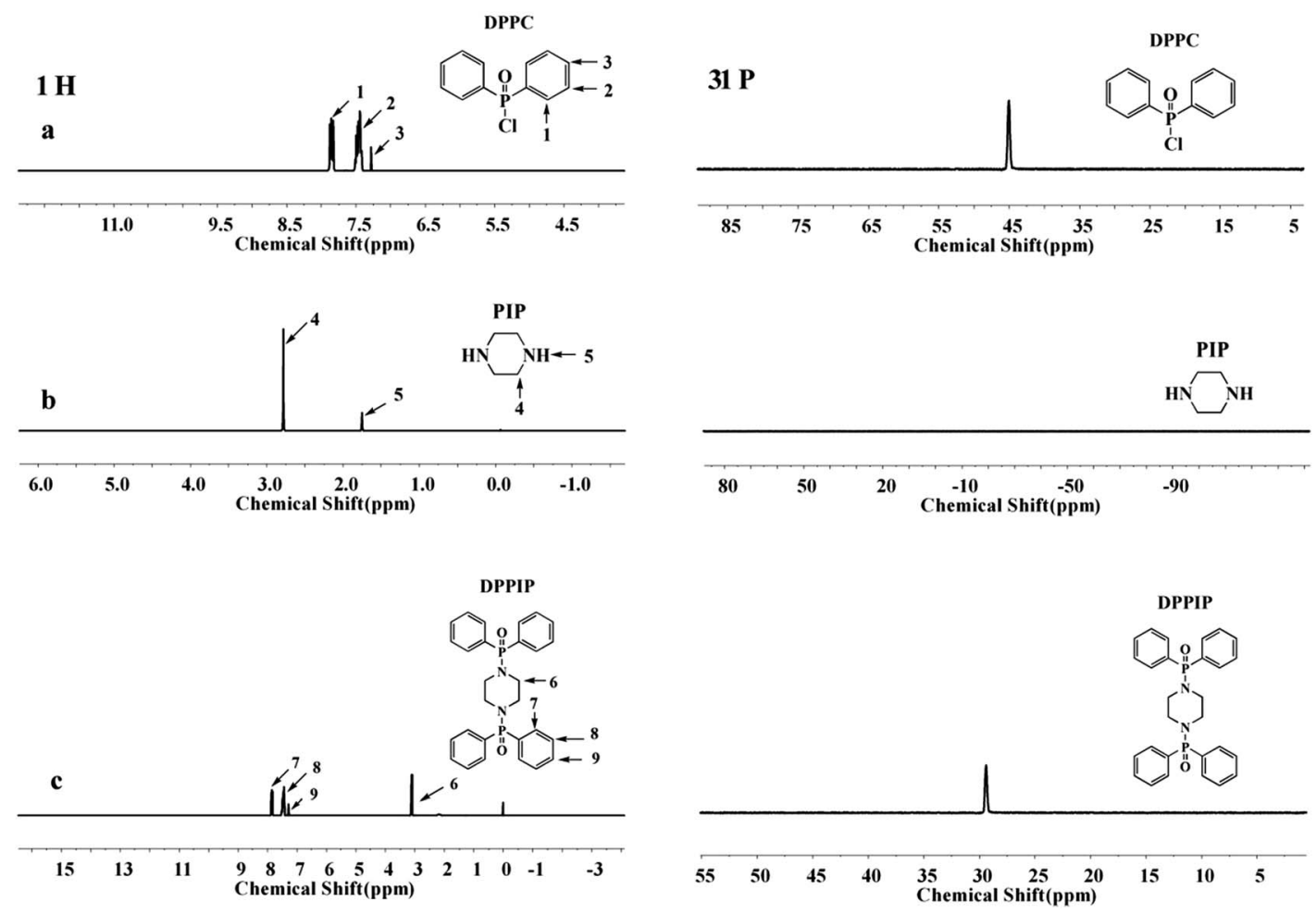

Fig. $2{ }^{1} \mathrm{H}-\mathrm{NMR}$ and ${ }^{31} \mathrm{P}-\mathrm{NMR}$ spectra of DPPC (a), PIP (b) and DPPIP (c).
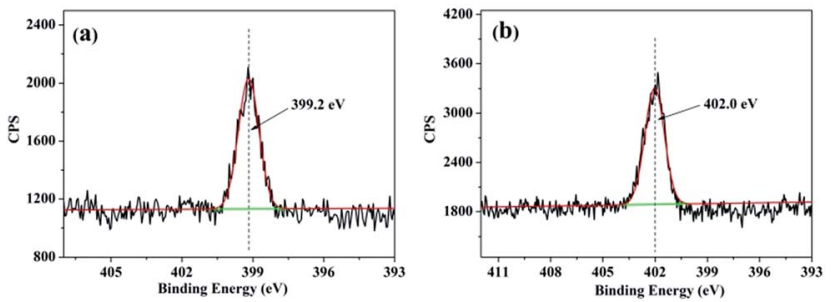

Fig. $3 \quad N_{1 s}$ spectra of PIP (a) and DPPIP (b)

Table 1 Water solubility of DPPIP, PA-APP, PP/DPPIP and PP/PA-APP

\begin{tabular}{llll}
\hline Sample & $\begin{array}{l}\text { Average water solubility } \\
(\mathrm{g} / 100 \mathrm{~mL} \text { of water })\end{array}$ & Sample & $\begin{array}{l}\text { Average weight } \\
\text { loss (wt\%) }\end{array}$ \\
\hline DPPIP & $<0.01$ & $\mathrm{PP} / 25 \mathrm{wt} \%$ DPPIP & $0.52 \pm 0.02$ \\
PA-APP & $=10.0$ & $\mathrm{PP} / 25 \mathrm{wt} \%$ PA-APP & $6.53 \pm 0.04$
\end{tabular}

Table 2 Thermogravimetric properties of PP, DPPIP and PP/DPPIP

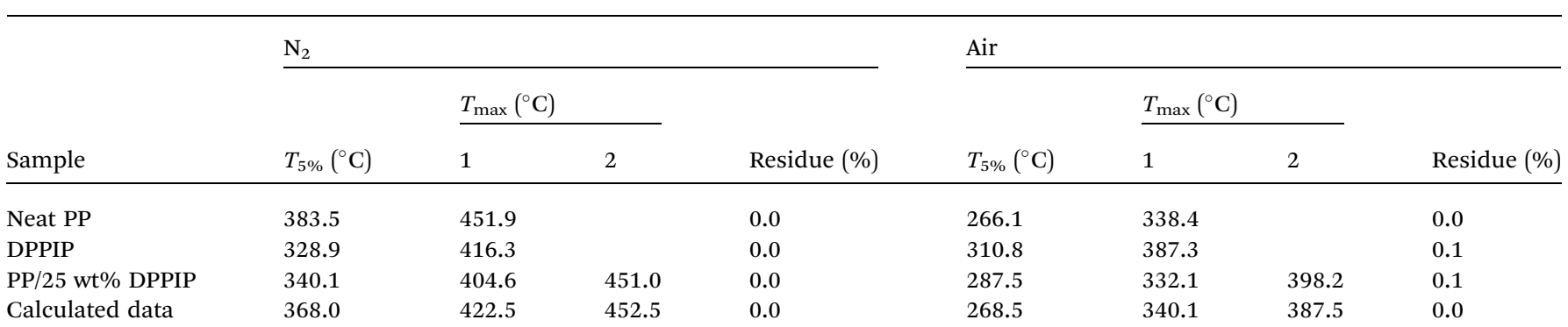

\subsection{Mechanical properties of flame-retardant PP composites}

The mechanical properties of PP/PA-APP and PP/DPPIP, including the tensile strength, elongation at break, and impact strength, are listed in Table 3. Compared with PP/PAAPP, PP/DPPIP with an equal amount of flame retardant had no apparent change in the impact strength, but both the tensile strength and elongation at break for PP/DPPIP increased. Therefore, it can be concluded that DPPIP is more conducive to the improvement of the mechanical properties of flameretardant PP than PA-APP.

\subsection{Flame retardancy}

The flame retardant performances of neat PP and PP/DPPIP before and after water-resistance tests were evaluated by LOI and UL-94 testing, and the corresponding data are listed in Table 4 . The two tests showed that neat PP is a flammable polymer. For PP/DPPIP, with an increasing amount of DPPIP, 

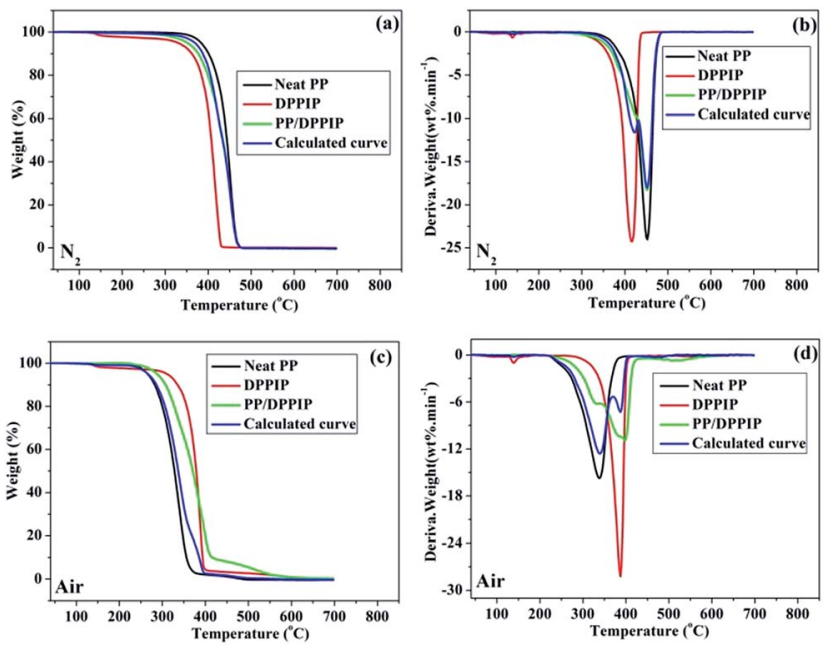

Fig. 4 TG and DTG curves of neat PP, DPPIP, and PP/25 wt\% DPPIP and the calculated curve of PP/25 wt\% DPPIP at a heating rate of $10{ }^{\circ} \mathrm{C} \min ^{-1}$ under $\mathrm{N}_{2}(\mathrm{a}, \mathrm{b})$ and air (c, d) atmosphere.

the LOI value increased from $18.0 \%$ (neat PP) to as high as $28.5 \%$ with the addition of $30 \mathrm{wt} \%$. Furthermore, with only 25 wt $\%$ addition of DPPIP, the flame-retardant PP can pass the UL-94 V0 rating and dripping is not observed. After the water treatment, the LOI value of flame-retardant PP decreased slightly, and when the addition of DPPIP was $25 \mathrm{wt} \%$, the LOI value decreased from $27.0 \%$ to $26.9 \%$, and this can still pass the UL-94 V0 rating. These results indicate that DPPIP is an effective flame retardant for PP, which could further meet the needs of practical application.

CC testing is an effective method to assess the combustion behavior of materials. The corresponding heat release rate (HRR), total heat release (THR), smoke production rate (SPR) and total smoke production (TSP) curves of neat PP and $\mathrm{PP} /$ $25 \mathrm{wt} \%$ DPPIP are shown in Fig. 5 and 6, and the corresponding data are presented in Table 5. As shown in Table 5, the time to ignition (TTI) of the neat PP (20 s) is far lower than that of PP/ DPPIP (58 s) due to the early decomposition of neat PP at the beginning of combustion, which is in accordance with the TGA results in air atmosphere. Therefore, the ignition-resistance of the composite is improved. With the addition of DPPIP, both the peak of the HRR (PHRR) and the THR of PP/25 wt\% DPPIP decreased compared with those of neat PP, especially for the PHRR, which decreased from $904.4 \mathrm{~kW} \mathrm{~m}^{-2}$ to $487.7 \mathrm{~kW} \mathrm{~m}^{-2}$
Table $4 \mathrm{LOI}$ and UL-94 results of neat PP and PP/DPPIP before and after water resistance testing

\begin{tabular}{|c|c|c|c|c|c|c|c|}
\hline \multirow{2}{*}{\multicolumn{2}{|c|}{$\begin{array}{l}\text { Component } \\
(\mathrm{wt} \%)\end{array}$}} & \multicolumn{3}{|c|}{ Not treated with water } & \multicolumn{3}{|c|}{ After water treatment } \\
\hline & & \multirow{2}{*}{$\begin{array}{l}\text { LOI } \\
(\%)\end{array}$} & \multicolumn{2}{|c|}{ UL-94 (3.2 mm) } & \multirow{2}{*}{$\begin{array}{l}\text { LOI } \\
(\%)\end{array}$} & \multicolumn{2}{|c|}{ UL-94 (3.2 mm) } \\
\hline $\mathrm{PP}$ & DPPIP & & Rating & Dripping & & Rating & Dripping \\
\hline 100 & 0 & 18.0 & NR & Yes & - & - & - \\
\hline 80 & 20 & 24.5 & NR & Yes & - & - & - \\
\hline 75 & 25 & 27.0 & V0 & No & 26.9 & V0 & No \\
\hline 70 & 30 & 28.5 & V0 & No & - & - & - \\
\hline
\end{tabular}
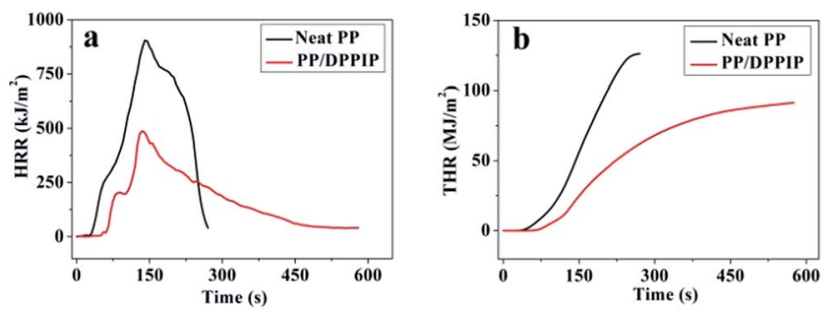

Fig. 5 HRR (a) and THR (b) plots of PP/25 wt\% DPPIP.
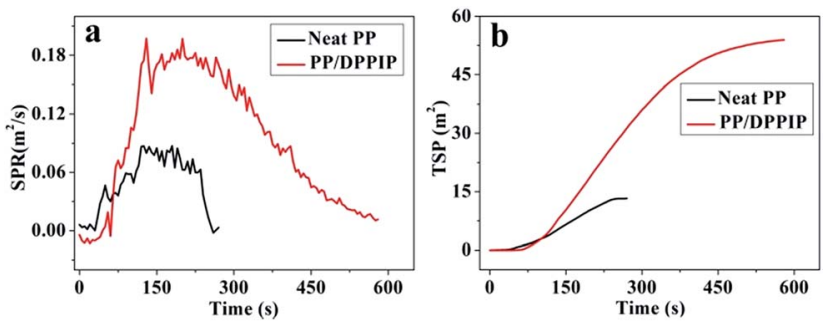

Fig. 6 SPR (a) and TSP (b) plots of PP/25 wt\% DPPIP.

with a reduction of $46.1 \%$ compared with that of $\mathrm{PP}$, indicating that the presence of DPPIP is favorable to suppress the heat release of neat $\mathrm{PP}$.

Based on the HRR curves, the fire growth rate (FGR) has been calculated to assess the fire hazard of the composite according to the following equation: ${ }^{21,22}$

$$
\mathrm{FGR}=\mathrm{PHRR} / t_{\mathrm{PHRR}}
$$

Table 3 Mechanical properties of PP/DPPIP and PP/PA-APP

\begin{tabular}{|c|c|c|c|c|}
\hline Sample & $\begin{array}{l}\text { Flame retardant } \\
(\mathrm{wt} \%)\end{array}$ & $\begin{array}{l}\text { Tensile strength } \\
(\mathrm{MPa})\end{array}$ & $\begin{array}{l}\text { Elongation at } \\
\text { break (\%) }\end{array}$ & $\begin{array}{l}\text { Impact strength } \\
\left(\mathrm{kJ} \mathrm{m}^{-2}\right)\end{array}$ \\
\hline \multirow[t]{3}{*}{ PP/DPPIP } & 20 & $38.8 \pm 0.3$ & $113.3 \pm 5.4$ & $1.83 \pm 0.04$ \\
\hline & 25 & $33.2 \pm 0.2$ & $87.5 \pm 6.3$ & $1.95 \pm 0.03$ \\
\hline & 30 & $29.5 \pm 0.3$ & $60.4 \pm 4.5$ & $1.98 \pm 0.04$ \\
\hline \multirow[t]{3}{*}{$\mathrm{PP} / \mathrm{PA}-\mathrm{APP}^{17}$} & 20 & $33.0 \pm 0.4$ & $92.4 \pm 8.2$ & $1.85 \pm 0.02$ \\
\hline & 25 & $30.6 \pm 0.3$ & $80.7 \pm 5.4$ & $1.92 \pm 0.03$ \\
\hline & 30 & $25.2 \pm 0.2$ & $43.2 \pm 5.5$ & $2.02 \pm 0.03$ \\
\hline
\end{tabular}


Table 5 CC data of neat PP and PP/25 wt\% DPPIP composites

\begin{tabular}{lll}
\hline Sample & Neat PP & PP/DPPIP \\
\hline TTI (s) & 20 & 58 \\
Peak HRR $\left(\mathrm{kW} \mathrm{m}^{-2}\right)$ & 904.4 & 487.7 \\
Time to PHRR (s) & 140 & 135 \\
FGR $\left(\mathrm{kW} \mathrm{m}^{-2} \mathrm{~s}\right)$ & 6.46 & 3.61 \\
THR $\left(\mathrm{MJ} \mathrm{m}{ }^{-2}\right)$ & 126.2 & 87.5 \\
Char residue (wt\%) & 0.5 & 1.1 \\
\hline
\end{tabular}

Generally, a lower FGR value indicates that the time to flashover is delayed. ${ }^{21}$ For PP/DPPIP, the FGR decreased from $6.46 \mathrm{~kW} \mathrm{~m}^{-2} \mathrm{~s}$ to $3.61 \mathrm{~kW} \mathrm{~m}^{-2} \mathrm{~s}$, which was decreased by $44.1 \%$ compared with that of neat PP. This result suggests that the introduction of DPPIP could significantly reduce the FGR of the PP composite, and consequently could significantly extend the time to escape in a real incident.

The SPR and TSP curves of neat PP and PP/DPPIP are presented in Fig. 6, and the corresponding data are summarized in Table 5. It is clear that the SPR and TSP of PP/DPPIP increased more significantly compared with those of neat PP. The results illustrate that PP/DPPIP could produce lots of smoke, and suggest that DPPIP generates gas-phase products during the decomposition.

The digital photos of the residues for neat PP and PP/DPPIP after the CC test are shown in Fig. 7, and the char residues are listed in Table 5. There is almost nothing left after burning, which is in accordance with the TGA results in air and $\mathrm{N}_{2}$ atmosphere, indicating that the gaseous phase production from the decomposition of DPPIP plays a positive role in the flame retardancy of PP. This can be described as follows: during combustion the decomposition of DPPIP occurs, then a lot of inflammable gas is produced which could dilute the oxygen concentration. As a consequence, the formation of inflammable gas should be the most important factor to achieve a better flame retardancy of PP.

\subsection{The flame retardant mechanism of DPPIP}

As mentioned above, to reveal the flame-retardant mechanism of DPPIP, the gaseous-phase activity of DPPIP is suggested. Thus, the gaseous phase products of DPPIP were investigated through TG-FTIR, and the FTIR spectra of the gaseous phase
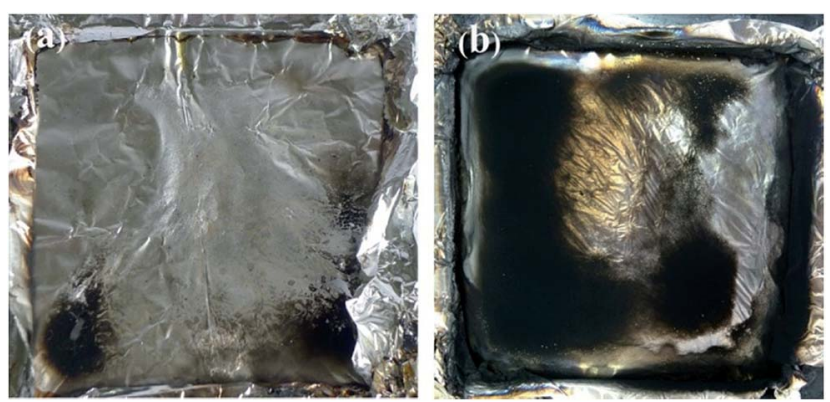

Fig. 7 Digital photographs of residues of neat PP (a) and PP/25 wt\% DPPIP (b) composites after the CC test. products of DPPIP during the thermal decomposition process under $\mathrm{N}_{2}$ atmosphere are shown in Fig. 8. The absorption peaks of the benzene rings at $3063.3 \mathrm{~cm}^{-1}$ and $695.1 \mathrm{~cm}^{-1}$ appeared at $328{ }^{\circ} \mathrm{C}$, and some nitrogen-containing and carbon-hydrogencontaining products were released at $328{ }^{\circ} \mathrm{C}$, as they presented the relevant characteristic absorption peaks at $2951.2 \mathrm{~cm}^{-1}\left(-\mathrm{CH}-,-\mathrm{CH}_{2}-,-\mathrm{CH}_{3}\right)$ and $1437.6 \mathrm{~cm}^{-1}(-\mathrm{NH}-$, $\left.-\mathrm{NH}_{2}\right)$. Meanwhile, the absorption peaks at $1246.2 \mathrm{~cm}^{-1}(\mathrm{P}=\mathrm{O})$, $1125.2 \mathrm{~cm}^{-1}$ and $960.6 \mathrm{~cm}^{-1}(\mathrm{P}-\mathrm{C})$ and $1125.2 \mathrm{~cm}^{-1}$ and $727.5 \mathrm{~cm}^{-1}(\mathrm{P}-\mathrm{N}-\mathrm{C})$ appeared. ${ }^{17,18,23}$ With increasing time, these absorbing peaks increased in intensity in the range from 328 to $418^{\circ} \mathrm{C}$. The results indicate that some non-flammable volatiles, such as benzene ring containing, nitrogen-containing, carbonhydrogen-containing and phosphorus-containing products are generated during the thermal degradation of DPPIP.

To further demonstrate the flame retardant mechanism of DPPIP, the prolyzed products of DPPIP were characterized with py-GC/MS measurement and the results are shown in Fig. 9, Table 6 and Scheme 2. It was found that the pyrolysis behavior of DPPIP is very complicated. When DPPIP is heated up to $600{ }^{\circ} \mathrm{C}$, a small molecule like $\mathrm{CO}_{2}$ is produced at first. With increasing time, more than twenty-three kinds of pyrolysis product were detected, which could be mainly summarized as three kinds of component. The first one was composed of piperazine and its derivatives, including the peaks at 2 (piperazine), 4 (pyrazine), 6 (3-ethylpyrrole), 7 (2-methylpyrazine), 8 ((E)-but-2-ene), 11 (2-methylimidazole) and 16 (4,4'-bipyridine), which were obtained from direct breaks of the piperazine ring and its rearrangement into decomposition segments. ${ }^{23,24}$ The

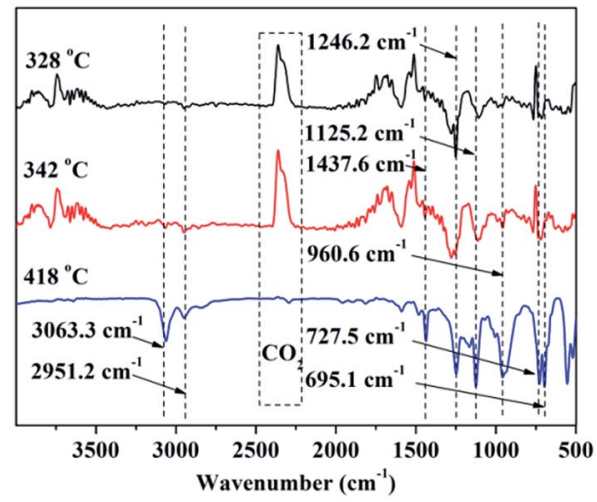

Fig. 8 FTIR spectra of the gaseous products of DPPIP during the thermal degradation under $\mathrm{N}_{2}$ atmosphere.

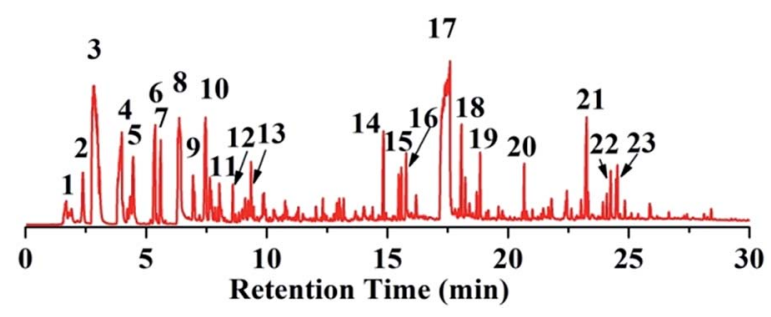

Fig. 9 Pyrograms of DPPIP 
Table 6 Compounds identified from the pyrograms of DPPIP

\begin{tabular}{|c|c|c|c|c|}
\hline Peak & Retention time (min) & $m / z$ & Compounds & Assigned structure \\
\hline 1 & 1.70 & 44 & Carbon dioxide & $\mathrm{O}=\mathrm{C}=\mathrm{O}$ \\
\hline 2 & 2.39 & 86 & Piperazine & \\
\hline 3 & 2.85 & 78 & Benzene & \\
\hline 4 & 3.99 & 80 & Pyrazine & \\
\hline 5 & 4.47 & 92 & Toluene & \\
\hline 6 & 5.38 & 95 & 3-Ethylpyrrole & \\
\hline 7 & 5.60 & 94 & 2-Methylpyrazine & \\
\hline 8 & 6.37 & 56 & $(E)$-But-2-ene & \\
\hline 9 & 6.94 & 104 & Ethenylbenzene & \\
\hline 10 & 7.46 & 110 & Phenylphosphineinhexane & \\
\hline 11 & 7.64 & 82 & 2-Methylimidazole & \\
\hline 12 & 8.03 & 124 & White phosphorus & $\mathrm{P}_{4}$ \\
\hline 13 & 9.35 & 107 & Methylphenylamine & \\
\hline 14 & 14.84 & 154 & Biphenyl & \\
\hline 15 & 15.58 & 168 & 2-Methylbiphenyl/4-methylbiphenyl & \\
\hline 16 & 15.78 & 156 & 4,4'-Bipyridine & \\
\hline 17 & 17.60 & 186 & Diphenylphosphin & \\
\hline 18 & 18.07 & 200 & Methyldiphenylphosphine & \\
\hline 19 & 18.84 & 214 & Ethyldiphenylphosphine & \\
\hline 20 & 20.66 & 201 & Diphenylphosphinoamine & \\
\hline
\end{tabular}


Table 6 (Contd.)

\begin{tabular}{|c|c|c|c|c|}
\hline Peak & Retention time (min) & $m / z$ & Compounds & Assigned structure \\
\hline 21 & 23.25 & 262 & Triphenylphosphine & \\
\hline 22 & 24.25 & 242 & Diphenylpropenyl phosphine oxide & \\
\hline 23 & 25.54 & 260 & Ethylenediamine phosphine oxide & \\
\hline
\end{tabular}

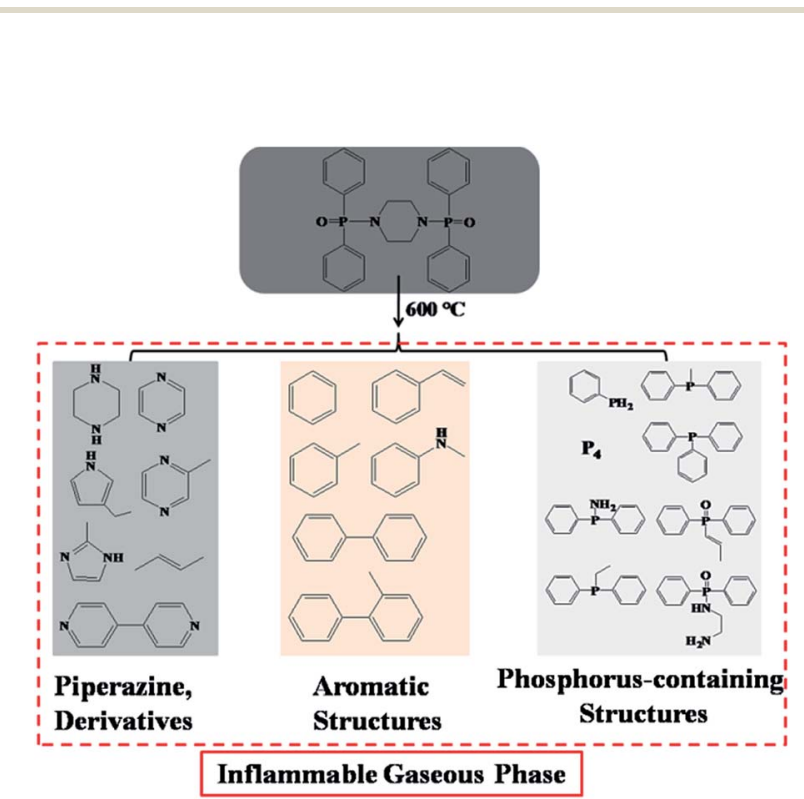

Scheme 2 Speculative pyrolysis products of DPPIP at $600^{\circ} \mathrm{C}$.

second one consisted of the compounds with aromatic structures (peaks at 3, 5, 9, 13,14 and 15), including the benzene ring and its derivatives. These aromatic derivatives occurred following the rupture of P-Ar. And last, lots of phosphoruscontaining structures were detected, corresponding to the peaks at $10,12,17,18,19,20,21,22$ and $23 .^{24-26}$ The phosphorus-containing structures could produce PO free radicals to capture active free radicals such as $\mathrm{H}$ and hydrocarbon free radicals during combustion. ${ }^{26}$ Generally, the result of PyGC/MS is consistent with those of the TG-FTIR and CC tests.

Based on the Py-GC/MS and FTIR-TG results, the effect of the gaseous phase on the flame retardation of PP is concluded as follows. With increasing temperature, lots of piperazine and its derivatives deriving from direct breaks of the piperazine ring and its rearrangement into decomposition segments are formed and released firstly; meanwhile, the P-Ar bonds break and aromatic structures are produced, which can further increase the amount of inflammable volatile gas. Besides, the rest of the P-Ar bonds continue to be degraded and rearranged, and some structures containing $\mathrm{P}$ are finally generated, which can capture active free radicals during the burning. ${ }^{26}$ Furthermore, the three kinds of inflammable volatile gases can reduce the oxygen content and capture active free radicals during combustion through the gaseous flame retardance mode, resulting in a superior flame-retardant efficiency of PP.

\section{Conclusion}

A mono-component flame retardant named DPPIP was prepared in this work. PP/DPPIP showed excellent thermal stability and water resistance: the thermal decomposition temperature of the PP/ $25 \mathrm{wt} \%$ DPPIP composite at $5 \mathrm{wt} \%$ weight loss is $287.5{ }^{\circ} \mathrm{C}$ under air atmosphere, and the weight loss was only about $0.52 \mathrm{wt} \%$ after the water treatment. Meanwhile, PP/DPPIP exhibited excellent flame-retardant efficiency. The LOI value of PP/DPPIP reached $27.0 \%$, and passed the V-0 rating at $25 \mathrm{wt} \%$ DPPIP. The CC data showed that the PHRR and low THR etc., especially the FGR, largely decreased compared with the corresponding values of neat PP. Furthermore, there was almost no char residue left. The study on the flame retardant mechanism of DPPIP indicated that lots of inflammable gaseous phase products are produced, such as piperazine and its derivatives, and phosphorus-containing structures, which finally lead to a better flame retardant performance for PP.

\section{Conflicts of interest}

There are no conflicts to declare.

\section{Acknowledgements}

This work was financially supported by the National Natural Science Foundation of China (grant number 51703011; 21504015; 51603018); Project of Jilin Provincial Department of Education (grant no. 2016329); Program of Jilin Provincial Department of Science \& Technology (grant no. 20170520125JH); and Natural Science Foundation of Fujian Province of China (grant number 2015J05094). 


\section{References}

1 K. C. Mai, Z. J. Li, Y. X. Qiu and H. M. Zeng, J. Appl. Polym. Sci., 2001, 80, 2617-2623.

2 J. Z. Liang, J. Q. Feng, C. P. Tsui, D. F. Liu, S. D. Zhang and W. F. Huang, Composites, Part B, 2015, 71, 74-81.

3 X. L. Chen, J. Yu, M. He, S. Y. Guo, Z. Luo and S. J. Lu, J. Polym. Res., 2009, 16, 357-362.

4 Q. H. Tang, R. J. Yang, Y. Song and J. Y. He, Ind. Eng. Chem. Res., 2014, 53, 9728-9737.

5 Y. Liu, C. L. Deng, J. Zhao, J. S. Wang, L. Chen and Y. Z. Wang, Polym. Degrad. Stab., 2011, 96, 363-370.

6 X. J. Lai, S. Tang, H. Q. Li and X. R. Zeng, Polym. Degrad. Stab., 2015, 113, 22-31.

7 H. L. Xie, X. J. Lai, R. M. Zhou, H. Q. Li, Y. J. Zhang, X. R. Zeng and J. H. Guo, Polym. Degrad. Stab., 2015, 118, 167-177.

8 G. Camino, N. Grassie and I. McNeill, J. Polym. Sci., Polym. Chem. Ed., 1978, 16, 95-106.

9 Y. L. Wang, X. M. Zhang, A. Li and M. Li, Chem. Commun., 2015, 51, 14801-14804.

10 M. C. Yewa, N. H. Ramli Sulong, M. K. Yew, M. A. Amalina and M. R. Johan, Prog. Org. Coat., 2015, 81, 116-124.

11 C. Y. Luo, J. D. Zuo, F. Q. Wang, Y. C. Yuan, F. Lin, H. H. Huang and J. Q. Zhao, Polym. Degrad. Stab., 2016, 129, 7-11.

12 Z. Z. Xu, J. Q. Huang, M. J. Chen, Y. Tan and Y. Z. Wang, Polym. Degrad. Stab., 2013, 98, 2011-2020.

13 K. Yang, M. J. Xu and B. Li, Polym. Degrad. Stab., 2013, 98, 1397-1406.

14 P. Y. Wen, X. M. Feng, Y. C. Kan, Y. Hu and R. K. K. Yuen, Polym. Degrad. Stab., 2016, 134, 202-210.
15 Z. J. Wang, Y. F. Liu and J. Li, ACS Sustainable Chem. Eng., 2017, 5, 2375-2383.

16 Z. B. Shao, C. Deng, Y. Tan, M. J. Chen, L. Chen and Y. Z. Wang, Polym. Degrad. Stab., 2014, 106, 88-96.

17 Z. B. Shao, C. Deng, Y. Tan, M. J. Chen, L. Chen and Y. Z. Wang, ACS Appl. Mater. Interfaces, 2014, 6, 73637370 .

18 Z. B. Shao, C. Deng, Y. Tan, L. Yu, M. J. Chen, L. Chen and Y. Z. Wang, J. Mater. Chem. A, 2014, 2, 13955-13965.

19 H. J. Lin, H. Yan, B. Liu, L. Q. Wei and B. S. Xu, Polym. Degrad. Stab., 2011, 96, 1382-1388.

20 C. L. Deng, C. Deng, J. Zhao, R. M. Li, W. H. Fang and Y. Z. Wang, Chin. J. Polym. Sci., 2015, 33, 203-214.

21 H. Breulet and T. Steenhuizen, Polym. Degrad. Stab., 2005, 88, 150-158.

22 Y. W. Yan, L. Chen, R. K. Jian, S. Kong and Y. Z. Wang, Polym. Degrad. Stab., 2012, 97, 1423-1431.

23 Thermal and fire performance of flameretarded epoxy resin: investigating interaction between resorcinol bis(diphenyl phosphate) and epoxin nanocomposites, ed. C. Katsoulis, E. Kandare, B. K. Kandola, T. R. Hull and B. K. Kandola, Royal Society of Chemistry, Cambridge, 2009, vol. 17, pp. 184-205.

24 L. P. Dong, C. Deng, R. M. Li, Z. J. Cao, L. Lin, L. Chen and Y. Z. Wang, RSC Adv., 2016, 6, 30436-30444.

25 L. P. Dong, C. Deng and Y. Z Wang, Polym. Degrad. Stab., 2017, 135, 130-139.

26 R. K. Jian, P. Wang, W. S. Duan, J. S. Wang, X. L. Zheng and J. B. Weng, Ind. Eng. Chem. Res., 2016, 55, 11520-11527. 